\title{
Resiliency in Elemental Computing
}

\author{
Joseph Hassoun \\ Director of Hardware Engineering and chip design, Element CXI
}

\begin{abstract}
This presentation describes a new reconfigurable architecture that lends itself to parallelizable applications such as SoftwareDefined Radio, while providing a new level of reliability, called resiliency. The architecture is called an Elemental Computing Array (ECA). At run time, code is dynamically placed into the ECA elements to work around defects on a device whether they were fabrication defects or came about later due to device wear out. Resiliency extends the useful lifetime of products and allows for graceful system degradation instead of catastrophic failure. The ECA combines four computational styles: sequential, data-flow, message-passing, and DMA in a rapidly-reconfigurable distributed system on a chip.

Also covered will be a description of the implementation of key algorithms of Orthogonal Frequency-Division Multiplexing (OFDM) on the new ECA reconfigurable architecture. OFDM is an essential technology for current and next generation standards such as $3 \mathrm{G} / 4 \mathrm{G}$, WiMax, and digital broadcasting standards. The implementation of these kinds of standards on the ECA architecture will be covered, as well as implementation of compute-intensive smart antenna techniques such as beamforming, MIMO, and maximum likelihood (ML) detection.
\end{abstract}

